\title{
Toward Robotic Needle Steering in Lung Biopsy: A Tendon-Actuated Approach
}

\author{
Louis B. Kratchman*, Mohammed M. Rahman, Justin R. Saunders, Philip J. Swaney \\ and Robert J. Webster III \\ Vanderbilt University, Department of Mechanical Engineering, Nashville, Tennessee, U.S.A.
}

\begin{abstract}
Needle tip dexterity is advantageous for transthoracic lung biopsies, which are typically performed with rigid, straight biopsy needles. By providing intraoperative compensation for trajectory error and lesion motion, tendondriven biopsy needles may reach smaller or deeper nodules in fewer attempts, thereby reducing trauma. An image-guided robotic system that uses these needles also has the potential to reduce radiation exposure to the patient and physician. In this paper, we discuss the design, workflow, kinematic modeling, and control of both the needle and a compact and inexpensive robotic prototype that can actuate the tendon-driven needle for transthoracic lung biopsy. The system is designed to insert and steer the needle under Computed Tomography (CT) guidance. In a free-space targeting experiment using a discrete proportional control law with digital camera feedback, we show a position error of less than $1 \mathrm{~mm}$ achieved using an average of 8.3 images $(\mathrm{n}=3)$.
\end{abstract}

Keywords: Steerable Needle, Robotic Surgery, Image-Guided Surgery, Lung Biopsy, CT-guided procedures.

\section{INTRODUCTION}

Percutaneous lung biopsy is a widely applied procedure for diagnosis of cancer and other pulmonary diseases. To monitor the biopsy needle as it is manually inserted towards a lesion, physicians typically use CT, CT fluoroscopy, or standard fluoroscopy. ${ }^{1}$ Several images are usually collected during insertion. If at any time the needle is misaligned with the target, the surgeon either manipulates the exposed rear portion of the needle to realign the tip (to compensate for small errors), or withdraws the needle to re-attempt the insertion. Each insertion attempt increases the risk of complications such as pneumothorax (lung collapsed by air) and hemothorax (lung collapsed by blood). Each attempt also increases radiation exposure to the patient and, in cases where the surgeon's hands remain in the imaging area, the surgeon as well. Achieving the desired target via this manual procedure is especially difficult for small, deep, and/or mobile targets. The challenge of hitting these targets often precludes percutaneous biopsy altogether, leaving the patient no alternatives other than to 1) undergo an invasive, open-lung biopsy, or 2) forgo treatment altogether in favor of waiting and hoping that nodules are not cancerous and do not grow over time.

To address some of the above shortcomings of manually-performed biopsy, robotic needle insertion has been proposed for percutaneous procedures (see e.g. Cleary ${ }^{2}$ ). Notably with respect to pulmonary applications, Xu et $\mathrm{al}^{3}{ }^{3}$ developed a CT fluoroscopy-guided robot that automatically inserts a biopsy needle into a pulmonary lesion while compensating for respiratory motion. Barrett et al. ${ }^{4}$ introduced a lightweight, patient-mounted, telerobotic needle insertion device made from inexpensive plastic parts. Ding et al. ${ }^{5}$ suggested a robotic approach for CTguided lung biopsies that used bevel steering to guide a standard biopsy needle, based on the nonholonomic steering model developed by Webster et al. ${ }^{6}$ All of these prior studies are promising steps toward clinical implementation of robotically controlled lung biopsy, demonstrating the feasibility and potential advantages of robotic assistance. However, the use of stiff, straight needles constrains the robot to mimic the human procedure (i.e., small corrections by needle base manipulation during insertion, with large corrections requiring withdrawal and reinsertion). It would be advantageous to be able to make larger corrections to needle trajectory during insertion, without requiring reinsertion. Steerable needles provide a potential solution.

Precurved needles have been previously developed for various soft tissue applications. Sze ${ }^{7}$ used a rigid precurved needle for transthoracic biopsy of targets obstructed by bone, blood vessels, or other tissues. Ebrahimi

\footnotetext{
* Corresponding Author. Email: lou.kratchman@vanderbilt.edu
} 
et al. ${ }^{8}$ introduced a needle consisting of a precurved stylet within a straight cannula, which used tip-tissue interaction forces to steer through soft tissue. A variety of other needle steering methods also exist. ${ }^{9,10}$ Most of these have been demonstrated in homogeneous soft tissue phantoms, and some in animal tissues. While the concept of applying needle steering in the lung has been suggested, ${ }^{2,11}$ to the authors' knowledge it has not yet been demonstrated in animal or human lung tissue. We hypothesize that porous lung tissue may be a significantly different environment than the homogeneous and fairly stiff rubber phantom tissues typically used for initial proof of concept needle steering studies. One other steering method that has been specifically designed to address this, and is capable of steering through free space as well as soft tissue, is the concentric tube approach. ${ }^{12-17}$ This technique has been suggested for use in the lung ${ }^{11}$ but not yet demonstrated.

An additional possible needle steering method, which has received less attention to date from the needle steering community than the methods mentioned above is tendon-based steering, which appears to be well-suited to use in transthoracic lung biopsy ${ }^{18}$ - and which we believe is particularly well suited for robotic implementation of the procedure. Tendon actuation is appealing for biopsy, since it permits straightforward control of needle curvature, as we discuss in subsequent sections of this paper. Mathis et al. ${ }^{18}$ introduced a manually-operated, tendon-driven, steerable biopsy needle specifically for lung biopsy, which was marketed for clinical use as the Seeker Steerable Biopsy Needle ${ }^{\mathrm{TM}}$ (PneumRx Inc.; Mountain View, CA). A small handle at the base of the needle is used in this design to pull tendons within a steering stylet, causing both the stylet and the cannula that surrounds it to bend in a direction corresponding to handle motion.

Our goal in this paper is to place tendon-driven, steerable biopsy needles under robotic control. The intent is to automatically perform intraoperative trajectory adjustments within soft intraparenchymal tissue, without the need to withdraw the needle. We believe that in addition to the previously articulated advantages of robotic transthorasic lung biopsy, a steerable needle will be capable of accurately reaching a small deep lesion with fewer withdrawals and reinsertions than would be necessary with a robotic system that manipulates a straight, rigid biopsy needle.

\section{CLINICAL WORKFLOW}

In the current clinical practice of CT-guided lung biopsy, the physician examines a preoperative scan to identify the target and desired entry position and orientation of the needle. The physician then locates this position with respect to fiducial markers placed on the chest wall outside the skin, and then manually inserts the needle ${ }^{19}$ (see Figure 1). During insertion, the physician iteratively inserts an incremental distance and then re-images, making minor course corrections by applying forces and torques to the back end of the needle.

When converting this to a robotically assisted procedure, we envision the physician manually orienting the robot using a lockable positioning arm that is fixed to the CT bed (possibly including a breakaway mechanism as
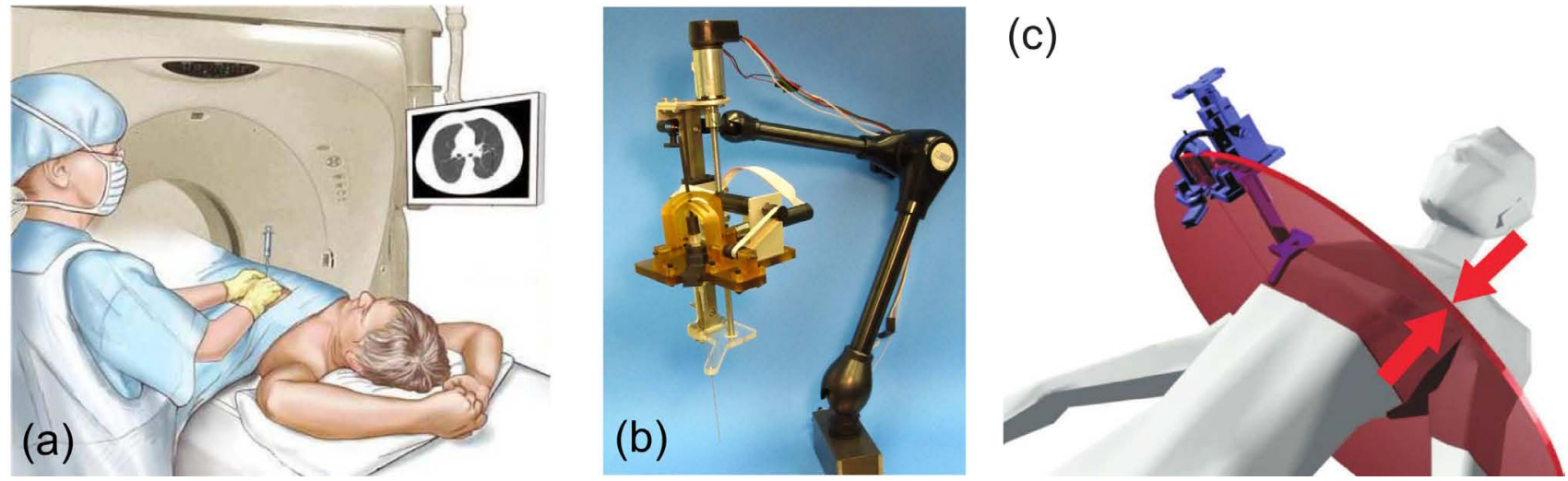

Figure 1. (a) In transthoracic lung biopsy the physician manually inserts a needle with image feedback. (Image adapted with minor modification from National Cancer Institute source image.) (b) A robot for lung biopsy can be mounted to a lockable positioning arm. (c) An illustration of the imaging plane, robot, and patient (figure adapted with modification from Blender Model Repository source image). 
in Shah et al. ${ }^{20}$ though this is not yet implemented on our system). After orienting the robot, the physician will retreat from the CT scanner for the duration of the targeting phase of the procedure, as the robot delivers the needle tip to the desired location indicated by the physician on CT images. When the needle tip has punctured the lesion, the physician will return to the patient to manually perform aspiration biopsy.

\section{NEEDLE DESIGN, WORKSPACE, AND KINEMATICS}

\subsection{Needle Design}

The Seeker biopsy needle is an example of a tendon-actuated needle marketed for lung biopsy. The needle can be curved by deflecting a handle mounted to its base (see Figure 2(a)). The handle pulls on tendons that run along the length of the central steering stylet, which is surrounded by an outer cannula (Figure 2(b-d)).
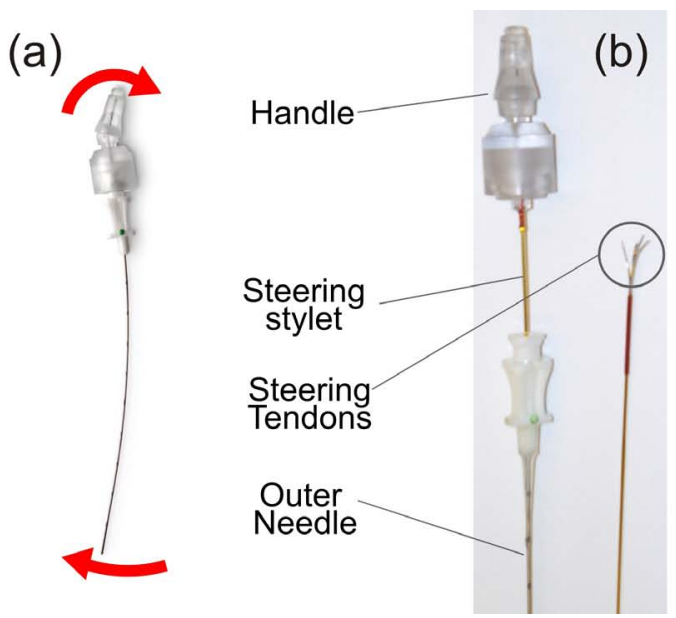

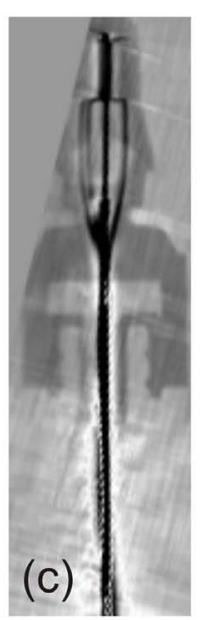

(d)

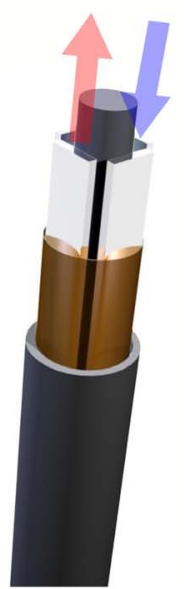

Figure 2. The Seeker needle is a tendon-actuated device. (a) It can be deflected by manipulating a handle at its base. (b) The complete needle consists of a stylet and outer cannula, where the stylet contains the tendons and generates the steering. On the right, a stylet with handle removed is shown. (c) A CT scan of an intact needle shows how tendons are routed. (d) A CAD illustration of the needle: the tendons surround a central flexible core, and are covered by a polymeric membrane to keep them in place.

\subsection{Needle Shape and Workspace}

To develop a controller for delivering the needle tip to a desired location, we require a kinematic model of needle shape. Ideally this model will describe how the shape of the needle (and hence its tip position) relates to the input degrees of freedom that the robot can control. Since the CT returns a set of planar images as illustrated in Figure 1(c), and we are interested in keeping the needle in a narrow range of CT scan slices, we will consider a planar model (even though the needle also has the capability to bend in and out of a plane). In this planar model (see Figure 3), the input degrees of freedom are the control handle angle in the plane $(\theta)$, and insertion distance $(\ell)$. We wish to relate these to the curvature of the deflected needle $(\kappa)$. It is well known in continuum robotics literature that unloaded, single-section, tendon-actuated robots approximate constant curvature, as long as the tendons are constrained to lie along a curve that is similar to the robot's central axis. ${ }^{21-23}$

We also verified experimentally that our needle assumes constant curvature (see Figures 3(b)-(d)). These experiments were performed using the purpose-built test platform shown in Figure 3(b). The platform consists of device for locking the handle angle to increments of $5^{\circ}$, mounted on a linear ball slide for adjusting the insertion depth of the needle. The tip of the needle protrudes through an acrylic plate attached to the front of the device, which constrains the needle to emerge orthogonally from the plate. To verify constant curvature, we obtained a set of eleven CT scans of the needle, deflected to angles from $-25^{\circ}$ to $25^{\circ}$, in $5^{\circ}$ increments. The scans were obtained with the Xoran xCAT ENT scanner (Xoran Technologies, Ann Arbor, Michigan, USA), which provides images with $0.4 \mathrm{~mm} \times 0.4 \mathrm{~mm}$ voxels. We segmented the needles from the images by thresholding, using ImageJ software, ${ }^{24}$ and manually identified points on the needle in pixel coordinates. 

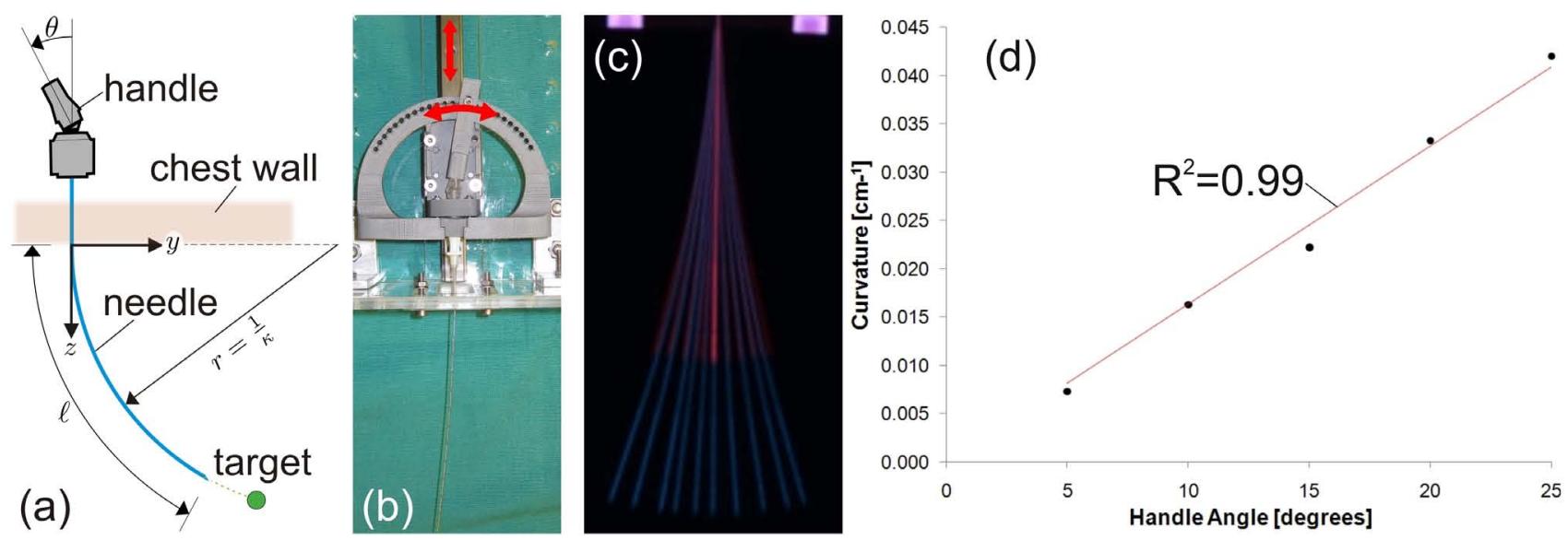

Figure 3. (a) A schematic of a tendon-driven steerable needle. (b) An apparatus for specifying handle angle and needle insertion distance prior to CT scanning. (c) The needle workspace: trajectories segmented from CT scans. (d) The linear experimental relationship found between the handle angle $\theta$ and needle curvature $\kappa$.

The curvatures listed in Figure 3(d) are those of circles tangent to the needle axis at the needle base, which also pass through experimentally determined needle tip positions. When plotted, these curves lie exactly on the needle shaft at all points along the needle. The linear relationship between joystick angle and needle curvature was determined to be,

$$
\kappa(\theta)=\frac{1}{r(\theta)}=\frac{9.36 \times 10^{-2}}{\mathrm{rad} \cdot \mathrm{cm}} \theta
$$

\subsection{Needle Kinematics}

For the constant curvature needle shown in Figure 3(a), we can identify the coordinates of any point on the needle shaft at arclength $s \in[0, \ell]$ using the geometric relationships,

$$
\begin{aligned}
& y=\frac{1-\cos \kappa s}{\kappa} \\
& z=\frac{\sin \kappa s}{\kappa} .
\end{aligned}
$$

If inverse kinematics is desired, given a desired tip location, one can solve these equations numerically for $\kappa$ and $s$, for example using Matlab's fsolve command, or any other suitable numerical method.

\section{ROBOT DESIGN}

To guide the tendon-driven biopsy needle described in Section 3 to a lung lesion, we have constructed the robot shown in Figure 4. The robot is designed to fit within the context of the surgical workflow described in Section 2. It controls three degrees of freedom: one for needle insertion or retraction, and two for applying curvature to the needle (one in the imaging plane and one orthogonal to it). The workspace of the needle controlled by this robot consists of a trumpet-shaped volume, a slice of which can be visualized in Figure 3(c) for a $100 \mathrm{~mm}$ insertion distance.

The base of the needle is attached to a platform that is raised or lowered to insert or retract the entire needle (see Figure 4). The platform is attached to a linear ball slide (THK; Schaumberg, Illinois), which ensures accurate linear motion. The platform is raised or lowered by a leadscrew (Haydon-Kerk; Waterbury, Connecticut), that is rotated by an A-Max series encoded DC motor (Maxon Precision Motors; Fall River, Massachusetts). The needle protrudes through a small hole in a plate attached to the bottom of the robot, which constraints the needle to be parallel to the axis of the linear slide.

To control needle curvature, two Maxon RE series DC motors are used to rotate the needle handle. The motors rotate two mutually-perpendicular arches, which each can rotate freely through an angle of $\pm 25^{\circ}$. A carbon fiber rod attached to the needle handle fits through through slots in both of the arches, such that 

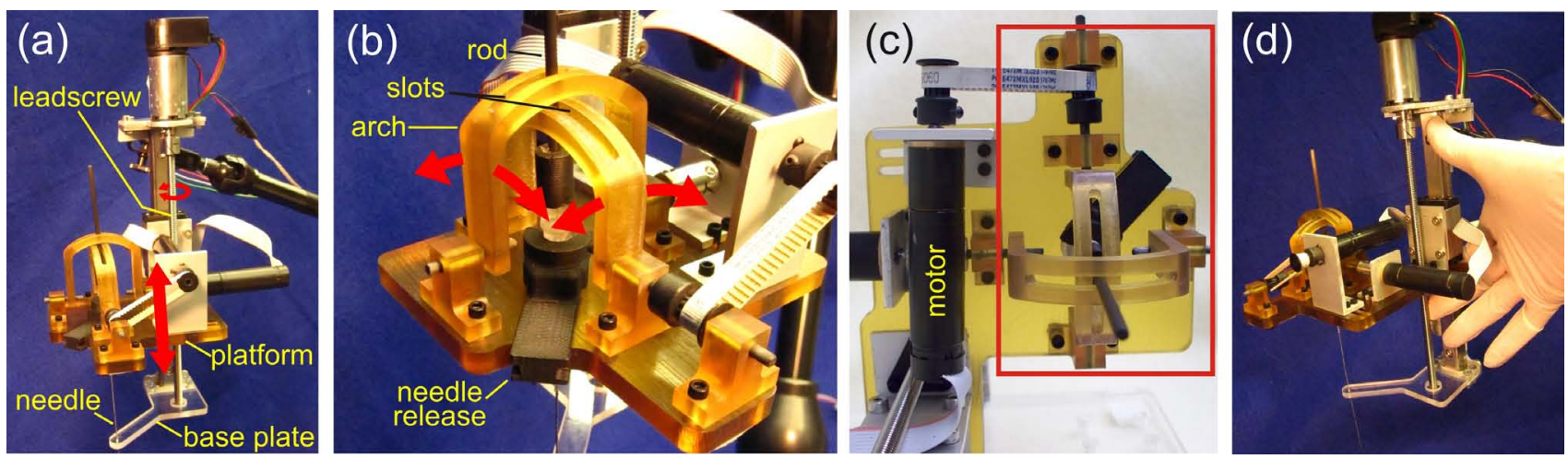

Figure 4. Images of the robot design. (a) A leadscrew accurately inserts or withdraws the platform holding the arches and needle. (b) The robot manipulates the handle by rotating two steering arches. (c) A view of the robot from above, with the box indicating the region through which CT images are acquired - it contains exclusively radiolucent materials to prevent image artifacts. (d) An image of the robot from the back.

coordinated motion of the arches can rotate the handle within a conical volume, to actuate the needle to desired positions within its $3 \mathrm{D}$ workspace.

Low-level PID control of each motor is accomplished using a Galil DMC 4080 motion controller (Galil Motion Control; Rocklin, California). To prevent the appearance of artifacts in CT images, no metal components other than the needle itself are contained within the portion of the robot that projects into the CT imaging area. The metal-free portion of the robot is shown in Figure 4(c).

\section{IMAGE-GUIDED CONTROL}

To guide the tip of the needle to a desired target, we use the following control algorithm. Given the desired target in image coordinates and a current CT scan identifying needle tip position, the algorithm determines the incremental needle handle adjustment $(\theta)$ and insertion distance $(\ell)$ to be applied before the next CT image is collected. To accomplish this, we convert the current needle tip position to its corresponding curvature and arc length $\left(\kappa_{i}, \ell_{i}\right)$, and the desired target position similarly to $\left(\kappa_{d}, \ell_{d}\right)$ via numerical solution of Equation 2 . We then use the following discrete proportional control laws,

$$
\begin{aligned}
& \theta_{i+1}=\theta_{i}+\alpha\left(\kappa_{d}-\kappa_{i}\right), \\
& \ell_{i+1}=\ell_{i}+\beta\left(\ell_{d}-\ell_{i}\right),
\end{aligned}
$$

where $\alpha$ and $\beta$ are gains that can be set as desired. To implement the control law, the handle is first deflected to $\theta_{i+1}$, then the needle is inserted to an arc length $\ell_{i+1}$, a new image is acquired, and the process is repeated until the target is achieved with the desired level of accuracy.

\section{EXPERIMENTAL RESULTS}

We performed a free-space targeting experiment using the robot described in Section 4 and the image-guided control algorithm described in Section 5. Each trial began with the needle inserted to a fixed starting depth of $3.3 \mathrm{~cm}$ beyond the base plate of the robot, which approximates the depth needed to pass through the chest wall and enter the lung. Three target points were selected at an insertion depth greater than $8 \mathrm{~cm}$ within the robot's workspace to simulate small, deep targets. Each insertion ended when the needle tip was measured to be within $1 \mathrm{~mm}$ of the desired target point. We used controller gains of $\alpha=15.71 \mathrm{rad} \cdot \mathrm{cm}$ and $\beta=0.5$ for each trial.

To measure the incremental position of the needle tip and to establish a space for defining targets, a regular grid of $1 \mathrm{~cm} \times 1 \mathrm{~cm}$ squares was inscribed on an acrylic sheet suspended approximately $3 \mathrm{~mm}$ beneath the needle, shown in Figure 5. As a substitute for a CT scanner, we attached a Kodak EasyShare Z1285 digital camera to a frame above the grid. The needle's tip location was determined relative to the physical grid in each camera image. 


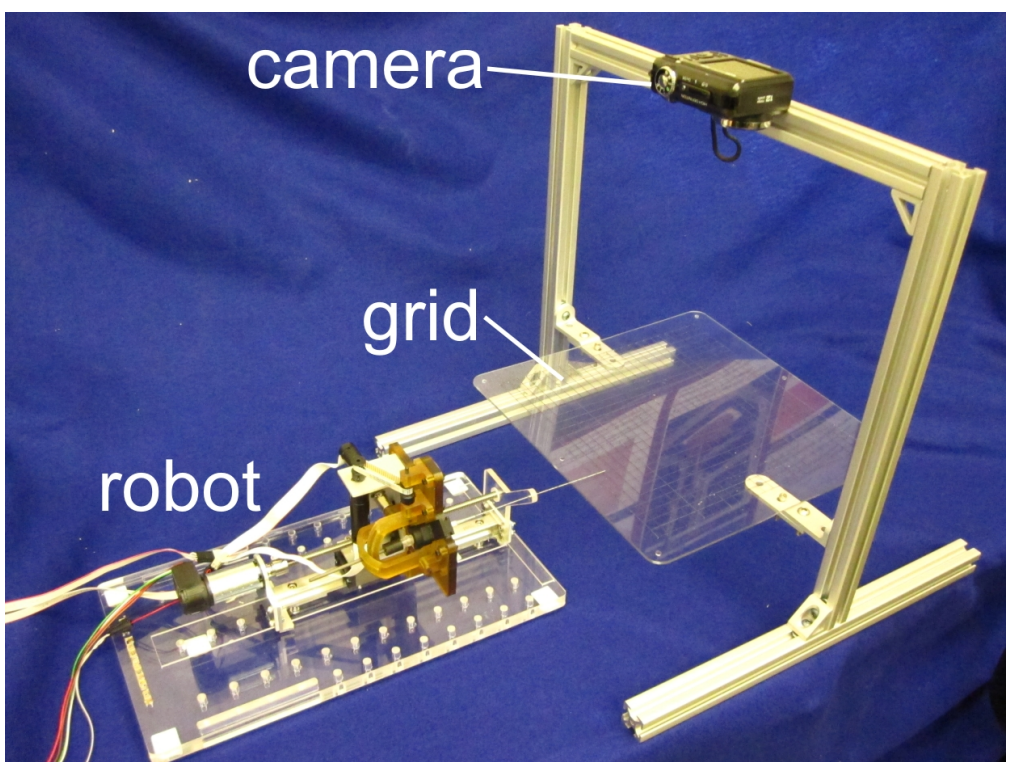

Figure 5. A digital camera was used to obtain position feedback during simulated targeting trials, as a substitute for a CT scanner.
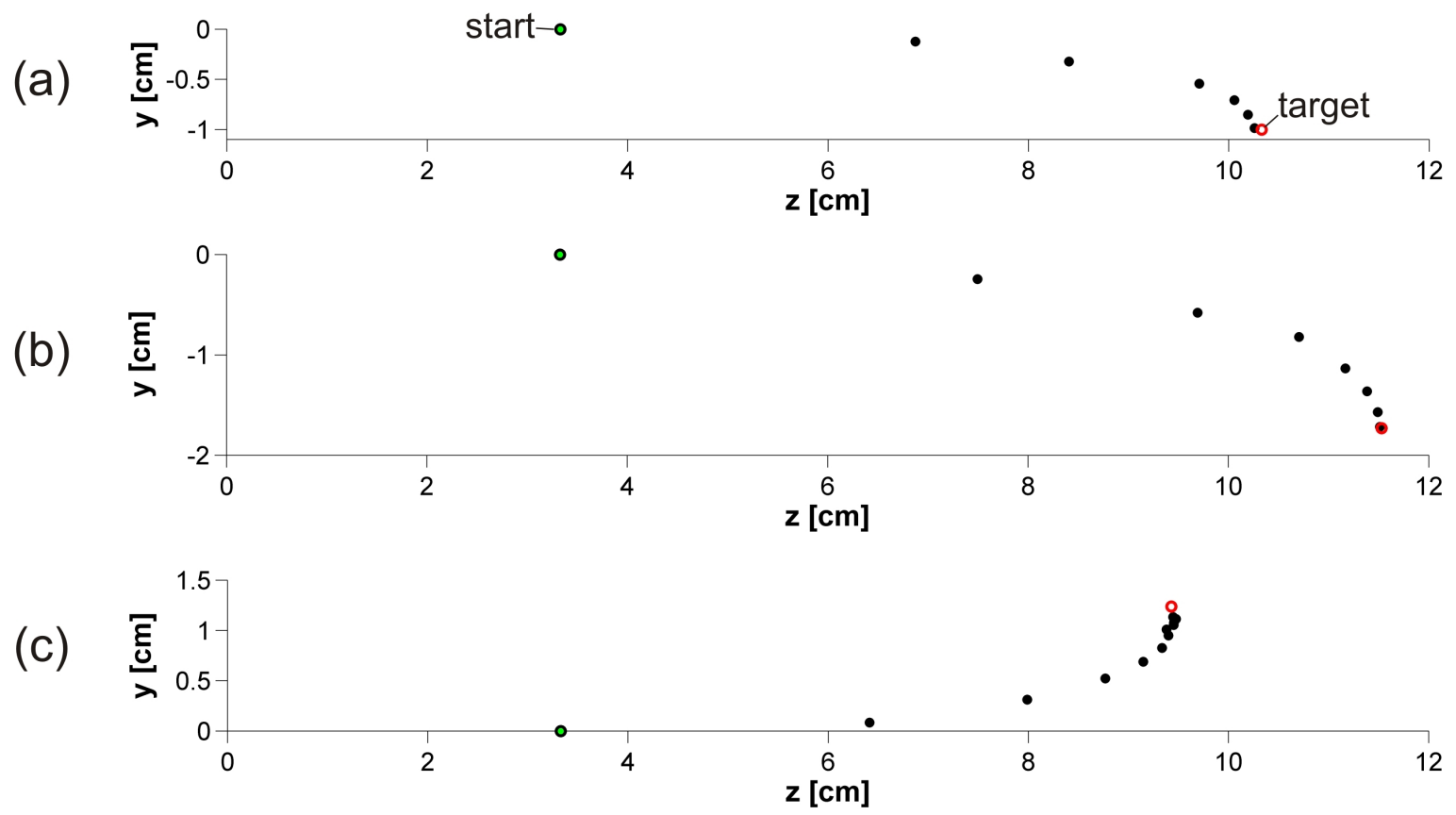

Figure 6. In each free space targeting trial, the needle successfully reached within $1 \mathrm{~mm}$ of each target point under automatic guidance.

Three experimental insertions were conducted. In the first, shown in Figure 6(a), the controller terminated in six steps at a target of $(\mathrm{z}=10.33 \mathrm{~cm}, \mathrm{y}=-1.01 \mathrm{~cm})$. In the second, shown in Figure 6(b), the target was $(\mathrm{z}=11.53 \mathrm{~cm}, \mathrm{y}=-1.73 \mathrm{~cm})$, and the needle required seven steps. The third trial, shown in Figure 6(c), required twelve steps to achieve a target of $(\mathrm{z}=9.43 \mathrm{~cm}, \mathrm{y}=-1.24 \mathrm{~cm})$. 


\section{CONCLUSION}

In this paper we have described the first system of which we are aware that is designed to robotically control a tendon-driven steerable needle. We discussed the design of one particular needle, which has been marketed for lung biopsy, and described its design, workspace, and kinematics. We then used it in several targeting experiments, demonstrating the use of image feedback to control tip position. We believe that a robot of this kind has the potential to improve accuracy and reduce radiation exposure for physicians and patients.

The next step in the development of this system will be targeting experiments similar to those we describe in this paper, but conducted using a porcine lung sample, in a CT scanner. Initial insertions into porcine lung lead us to expect that the needle will retain its circular shape (meaning that the modeling and control framework in this paper can be directly applied), but with a lower curvature than it achieves in free space. We also note that if higher curvature is desired, it may be possible to redesign the outer cannula - in the Seeker needle, the cannula is stiff and limits the maximum achievable curvature. In summary, we believe that the robotic design, modeling, and control framework described in this paper key steps toward clinical application of tendon-actuated steerable needles.

\section{ACKNOWLEDGMENTS}

The authors wish to acknowledge Grégoire Blachon, Caleb Rucker and Ray Lathrop for assistance with robot construction and targeting experiments, Conor Walsh for generously sharing his supply of biopsy needles, and Josh Heck for sharing his clinical insight.

\section{REFERENCES}

1. S. Gupta and D. Madoff, "Image-guided percutaneous needle biopsy in cancer diagnosis and staging," Techniques in Vascular and Interventional Radiology 10(2), pp. 88-101, 2007.

2. K. Cleary, "CT Fluoroscopy-Guided Robotically-Assisted Lung Biopsy," in Proc. of SPIE, 6141, pp. 61411L-1-8, 2006.

3. S. Xu, G. Fichtinger, R. Taylor, and K. Cleary, "3D motion tracking of pulmonary lesions using CT fluoroscopy images for robotically assisted lung biopsy," in Proc. of SPIE, 5367, pp. 394-402, 2004.

4. S. Barrett, N. Hanumara, C. Walsh, A. Slocum, R. Gupta, and J. Shepard, "A remote needle guidance system for percutaneous biopsies," Proceedings of the ASME International Design Engineering Conferences , 2005.

5. J. Ding, D. Stoianovici, D. Petrisor, P. Mozer, R. Avila, L. Ibanez, W. Turner, D. Yankelvitz, E. Wilson, F. Banovac, and K. Cleary, "Medical needle steering for lung biopsy: Experimental results in tissue phantoms using a robotic needle driver," in IEEE International Conference on BioInformatics and BioEngineering, pp. 1-5, 2008.

6. R. Webster, J. Kim, N. Cowan, G. Chirikjian, and A. Okamura, "Nonholonomic modeling of needle steering," The International Journal of Robotics Research 25(5-6), p. 509, 2006.

7. D. Sze, "Use of curved needles to perform biopsies and drainages of inaccessible targets," Journal of Vascular and Interventional Radiology 12(12), pp. 1441-1444, 2001.

8. R. Ebrahimi, S. Okazawa, R. Rohling, and S. Salcudean, "Hand-held steerable needle device," Medical Image Computing and Computer-Assisted Intervention-MICCAI 2003 , pp. 223-230, 2003.

9. N. Abolhassani, R. Patel, and M. Moallema, "Needle insertion into soft tissue: A survey," Medical Engineering \& Physics 29, p. 413431, 2007.

10. R. J. Webster III, Design and Mechanics of Continuum Robots for Surgery. Mechanical engineering, Johns Hopkins University, Baltimore, MD, December 2007. PhD Thesis.

11. L. A. Lyons, R. J. Webster III, and R. Alterovitz, "Planning active cannula configurations through tubular anatomy," IEEE International Conference on Robotics and Automation, pp. 2082-2087, 2010.

12. R. Webster, J. Romano, and N. Cowan, "Mechanics of precurved-tube continuum robots," Robotics, IEEE Transactions on $\mathbf{2 5}(1)$, pp. 67-78, 2009.

13. D. Rucker, B. Jones, and R. Webster III, "A Geometrically Exact Model for Externally Loaded ConcentricTube Continuum Robots," Robotics, IEEE Transactions on 26(5), pp. 769-780, 2010. 
14. P. Dupont, J. Lock, B. Itkowitz, and E. Butler, "Design and control of concentric-tube robots," Robotics, IEEE Transactions on 26(2), pp. 209-225, 2010.

15. C. Walsh, J. Franklin, A. Slocum, and R. Gupta, "Material selection and force requirements for the use of pre-curved needles in distal tip manipulation mechanisms," J. Med. Devices 4, p. 027536, 2010.

16. E. C. Burdette, D. C. Rucker, P. Prakash, C. J. Diederich, J. M. Croom, C. Clarke, P. J. Stolka, T. Juang, E. M. Boctor, and R. J. Webster III, "The ACUSITT ultrasonic ablator: The first steerable needle with an integrated interventional tool," Proc. of SPIE , 2010.

17. M. Terayama, J. Furusho, and M. Monden, "Curved multi-tube device for path-error correction in a needleinsertion system," International Journal of Medical Robotics and Computer Assisted Surgery 3(2), pp. 125$134,2007$.

18. M. Mathis, D. Thompson, B. Addis, and D. Yankelvitz, "Steerable device for accessing a target site and methods," Nov. 23 2005. US Patent App. 11/286,445.

19. E. Moore, "Percutaneous Lung Biopsy: An Ordering Clinician's Guide to Current Practice," in Seminars in respiratory and critical care medicine, 29(4), pp. 323-334, New York: Thieme Medical Publishers, c1994-, 2008.

20. S. Shah, A. Kapoor, J. Ding, P. Guion, D. Petrisor, J. Karanian, W. Pritchard, D. Stoianovici, B. Wood, and K. Cleary, "Robotically assisted needle driver: Evaluation of safety release, force profiles, and needle spin in a swine abdominal model," International Journal of Computer Assisted Radiology and Surgery 3(1-2), pp. $173-179,2008$.

21. C. Li and C. D. Rahn, "Design of continuous backbone, cable-driven robots," ASME Journal of Mechanical Design 124(2), pp. 265-271, 2002.

22. M. W. Hannan and I. D. Walker, "Kinematics and the implementation of an elephant's trunk manipulator and other continuum style robots," Journal of Robotic Systems 20(2), pp. 45-63, 2003.

23. R. J. Webster III and B. A. Jones, "Design and kinematic modeling of constant curvature continuum robots: A review," International Journal of Robotics Research 29(13), pp. 1661-1683, 2010.

24. M. Abramoff, P. Magelhaes, and S. Ram, "Image processing with ImageJ," Biophotonics international 11(7), pp. 36-42, 2004. 